\title{
Dense Focal Plane Arrays for Pushbroom Satellite Radiometers
}

\author{
O. A. Iupikov ${ }^{1}$, M. V. Ivashina ${ }^{1}$, K. Pontoppidan ${ }^{2}$, P. H. Nielsen², C. Cappellin ${ }^{2}$, N. Skou ${ }^{3}$, S. S. Søbjærg ${ }^{3}$, \\ A. Ihle ${ }^{4}$, D. Hartmann ${ }^{4}$, K. v. t Klooster \\ ${ }^{1}$ Signals and Systems Department of the Chalmers University of Technology, Gothenburg, Sweden, \\ e-mail: oleg.iupikov@chalmers.se, marianna.ivashina@chalmers.se \\ ${ }^{2}$ TICRA, Copenhagen, Denmark, e-mail: cc@ticra.com, kp@ticra.com,phn@ticra.com \\ ${ }^{3}$ DTU-Space, Technical University of Denmark, Kgs. Lyngby, Denmark, e-mail: ns@space.dtu.dk, sss@ space.dtu.dk \\ ${ }^{4}$ HPS GmbH, Munich, Germany, e-mail: ihle@hps-gmbh.com, hartmann@hps-gmbh.com \\ ${ }^{5}$ European Space Research and Technology Centre, Noordwijk, The Netherlands, e-mail: Kees.van.t.Klooster@esa.int
}

\begin{abstract}
Sammendrag-Performance of a dense focal plane array feeding an offset toroidal reflector antenna system is studied and discussed in the context of a potential application in multi-beam radiometers for ocean surveillance. We present a preliminary design of the array feed for the 5-m diameter antenna at X-band. This array is optimized to realize high antenna beam efficiency $(\sim 95 \%)$ over a wide scan range $\left( \pm 20^{\circ}\right)$ with very low side-lobe and cross-polarization levels.
\end{abstract}

Index Terms-reflector antenna feeds, multi-beam antennas, microwave radiometry.

\section{INTRODUCTION}

Recent advances in phased-array antenna technologies and low-cost active electronic components open up new possibilities for designing Earth observation instruments, in particular those used for radiometric measurements. Nowadays, two design concepts of microwave radiometers are in use: "pushbroom" and "whisk-broom" scanners [1]. Push-broom scan-

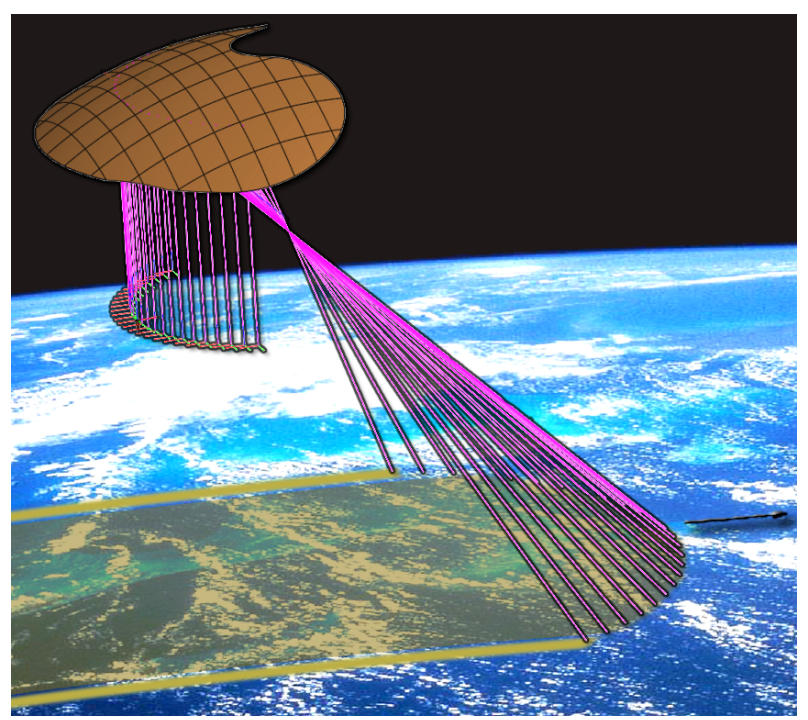

Figur 1. Operational principle of a push-broom microwave radiometer, which includes an off-set toroidal reflector antenna fed with a multi-beam focal plane array of horns arranged perpendicular to the flight direction of the spacecraft. Different areas of the ocean-surface are scanned as the spacecraft flies forward ners have an important advantage over whisk-broom scanner in providing larger field-of-view with higher sensitivity, owing to the fact that these systems can look at a particular area of the ocean for a longer time with multiple simultaneous beams. This concept is illustrated on Fig. 1, where one can see several beams, arranged perpendicular to the flight direction of the spacecraft. However, the drawback of pushbroom designs based on conventional focal plane arrays (FPAs) of horns in one-horn-per-beam configuration [2] or clusters with simplistic beamforming [3] - is the varying sensitivity. This variation occurs due to the difference between the scanned beams (as these are formed by different horns/clusters) and their large separation on the ocean surface, as the result of the large separation between the horns.

This drawback may be significantly reduced by employing dense FPAs, i.e. phased-array feeds consisting of many electrically small antenna elements, with advanced beamforming [4]. This technology has been extensively studied during the last decade in the radio astronomy community, and several telescopes are currently being equipted with dense FPAs [5] [7]. While those systems aim to provide the scan range of about $5-10$ beamwidths, for applications as herein considered, the desired scan range (swath range of the radiometer) is one order of magnitude larger [8]. Therefore, to achieve this performance, more complex designs of the reflector optics and FPA are required. For push-broom radiometers, various optics concepts have been investigated [2], and the optimum solution has been found to be an offset toroidal single reflector antenna, such as illustrated on Fig. 1. This reflector structure is rotationally symmetric around a vertical axis, and thus is able to cover a wide swath range. However, its aperture field exhibits significant phase errors due to the non-ideal (paraboloid) surface of the reflector - as compared to that of classical paraboloids. The phase errors cause degradation of the antenna beam efficiency and increase the side-lobe and cross-polarization levels. These degradations, in turn, limit the radiometer characteristics (such as the minimum distance to coast at which the measurement data remains usable) as well as worsen the situation with Radio Frequency Interference (RFI) that is problematic at many radiometer bands [9]. 
The purpose of this work is, therefore, (i) to determine to what extent the performance-limiting factors of push-broom radiometers can be reduced by using dense FPAs with advanced beamforming; and (ii) what is the minimum complexity of the FPA design (size, number of elements) that is required for meeting the instrument specifications at which future radiometers aim [8]. To address these questions, we have created an initial numerical model of the array that is based on the MoM-CBFM-model in [4]; the elements of this array represent tapered-slot antennas, as designed for the FPA system in [5]. To perform the parametric study, we have implemented this model for different array sizes and inter-element separation distances varying from 0.5 to 1 wavelength. For the evaluation of the radiometer characteristics, two beamforming methods have been considered that aim to optimize the beam efficiency with the minimum distance to land and cross-polarization loss.

\section{Antenna ReQuirements}

In February 2013 the ESA contract 4000107369-12-NLMH was awarded the team consisting of TICRA, DTU-Space, HPS and Chalmers University. The group comprises experts in reflector antennas design and analysis, passive microwave radiometry, mechanical and thermal analysis of ultra-light mesh reflector technology, and radio astronomy with the knowledge of dense focal plane arrays designs. As a part of this activity, we perform a preliminary design study of a pushbroom antenna, as shown on Fig. 1 with conventional FPAs of horns, as well as novel dense FPAs with active beamforming.

To identify the best design for the targeted application, we use the list of antenna system requirements that has been derived in [8], based on the the instrument specifications for accurate sea surface temperature and ocean vector wind measurements. This list includes the values for the required half-power beamwidth (and corresponding footprint on the sea-surface), acceptable cross-polarization power, as well as the minimum distance to coast at which the radiometer stops working correctly. It can be shown that in order to meet the requirements for radiometer characteristics (maximum allowed error of the measured sea brightness temperature $\Delta T<0.25 \mathrm{~K}$ and the distance to coast $<15 \mathrm{~km}$ ) the power incident on the land must be less than $0.14 \%$ of the total power hitting the Earth. This requirement leads to stringent constraints on both the side-lobe and cross-polarization levels of the antenna beams.

At present, the pushbroom antenna which can satisfy these requirements is a torus antenna with projected aperture of $5 \mathrm{~m}$, $\left( \pm \mathbf{2 0}^{\circ}\right)$ scan, the focal length to diameter ratio $\mathrm{f} / \mathrm{D}=1$ and swath of $600 \mathrm{~km}$. This antenna has been designed by TICRA; it achieves the swath on the Earth equal to $600 \mathrm{~km}$, assuming the satellite altitude above the Earth of $817 \mathrm{~km}$ and the incidence angle of $53^{\circ}$. This antenna should operate at C-band $(6.9 \mathrm{GHz}), \mathrm{X}$-band $(10.65 \mathrm{GHz})$ and $\mathrm{Ku}$-band $(18.7 \mathrm{GHz})$ with bandwidth $300 \mathrm{MHz}, 100 \mathrm{MHz}$ and $200 \mathrm{MHz}$, respectively. The analysis in this paper is performed at X-band only.

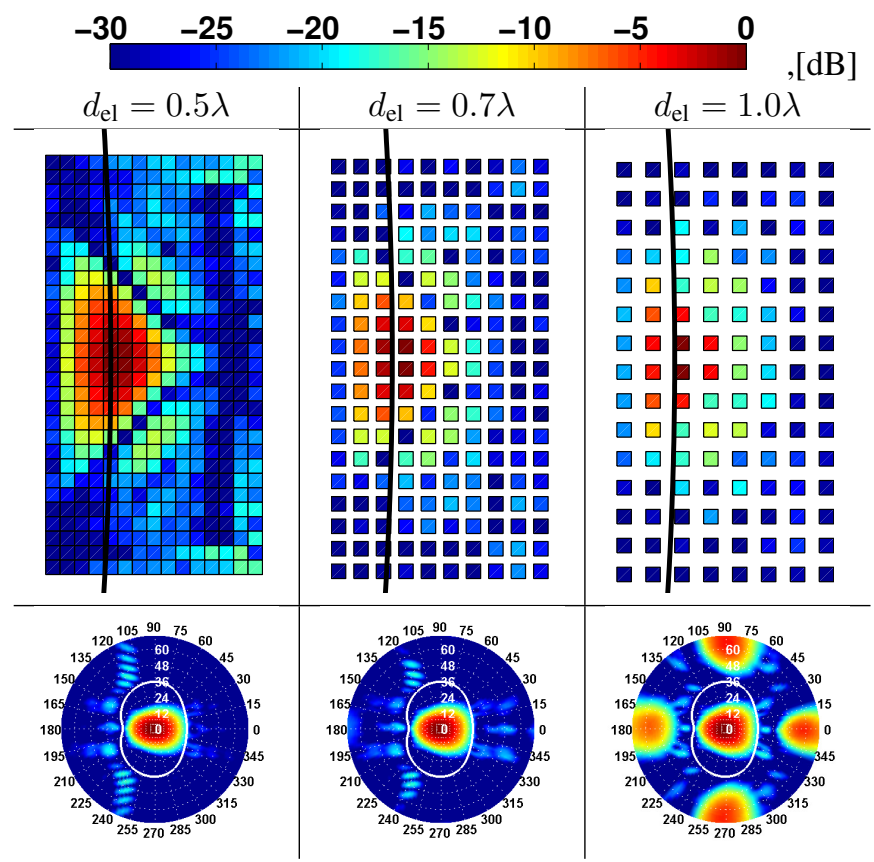

Figur 2. Effect of the inter-element separation distance $d_{\mathrm{el}}$ on (top) the optimized amplitude weights of the FPA sub-array elements for the centre beam, as determined for the customized beamformer maximizing the beam efficiency (@ - 20 dB) with constraints on the side-lobe and cross-polarization levels towards the Earth, and (bottom) the resultant illumination patterns of the reflector antenna. The array size is fixed to $L_{\mathrm{x}} \times L_{\mathrm{y}}=7 \lambda \times 14 \lambda$.

\section{FPA-SYSTEM DESIGN}

\section{A. Antenna array model}

As a starting point of the design procedure, we have considered a sub-array for the centre beam. The selected initial model of this sub-array represents a dual-polarized antenna array consisting of $15 \times 29 \times 2$ interconnected tapered-slot antenna elements with the inter-element distance varying from $0.5 \lambda$ to $1.0 \lambda$. This model is based on the MoM-CBFM model of the $8 \times 9 \times 2$ element array in [4]. To reduce the computational time for our parametric studies, we have simplified this original model by assuming that all embedded element patterns are identical to that of the central element of the finite array. The sub-array embedded element patterns have been imported into the reflector antenna software GRASP10 to compute the secondary embedded element patterns (after reflection from the dish), which, in turn, have been used to simulate the overall receiving system (according to the procedure in [4]), and to optimize its beamforming weights. It is worth mentioning that this analysis and optimization procedure accounts for the effects of the array mutual coupling, elements loading, as well as the signal and noise properties of the terminating amplifiers. The later effects have not been considered yet and are left for the future work.

\section{B. Beamforming algorithms}

For this study, we have implemented two types of signalprocessing beamforming algorithms: (i) standard maximum directivity beamformer (see Eq.3 in [4]), which is equivalent 


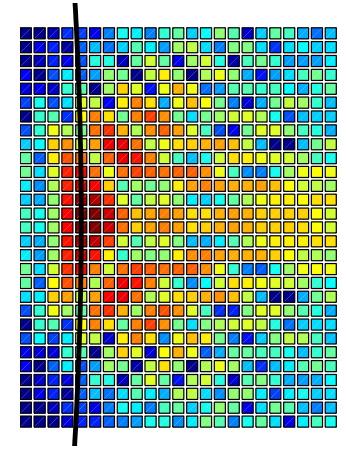

(a) CFM-BF weights
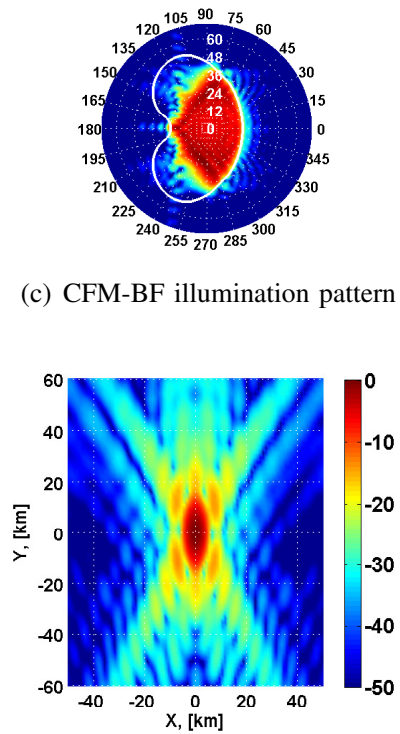

(e) CFM-BF footprint (c) CFM-BF illumination pattern

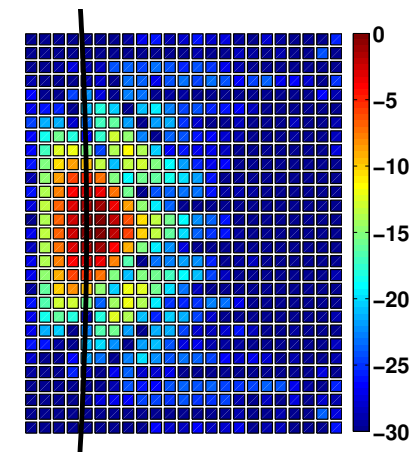

(b) Customized-BF weights

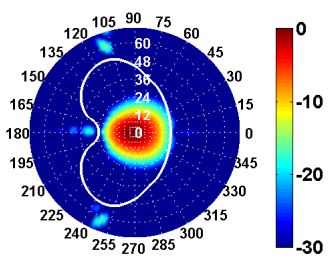

(d) Customized-BF illumination pattern

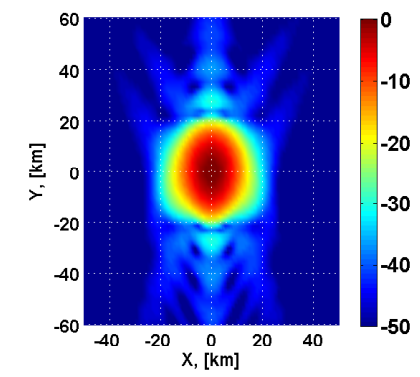

(f) Customized-BF footprint
Figur 3. Comparison of two beamforming algorithms for the FPA sub-array for the centre beam: $(a, b)$ the array element amplitude weight coefficients for the CFM beamformer (CFM-BF) and customized beamformer (Customized$\mathrm{BF})$, where each block represents an element and the black line shows the focal line of the torus reflector, and the the corresponding $(\mathrm{c}, \mathrm{d})$ reflector aperture illumination patterns and $(e, f)$ footprint patterns on the sea-surface.

(under certain conditions) to the Conjugate Field Matching (CFM) beamformer, as commonly applied to conventional FPAs of horns; and (ii) customized beamformer that has been formulated so as to maximize the beam efficiency (within the $-20 \mathrm{~dB}$ area), subject to constraints on the total radiated power towards the coastal region. The latter approach is expected to lead to the optimal radiometer performance in terms of the minimum distance to land, minimum cross-polarization and side-lobe levels, and thus improved resistance to RFI.

Figure 3 illustrates the examples of the optimized weight coefficients for the sub-array elements for the CFM and constrained beamformers, where the corresponding aperturefield distributions and footprint patterns of the antenna are presented below. As expected, the CFM beamformer leads to the highest directivity (the larger area of the reflector aperture that is illuminated efficiently), as compared to that of the constrained beamformer. On the other hand, the latter has a significantly improved shape of the footprint and much lower side-lobe level.
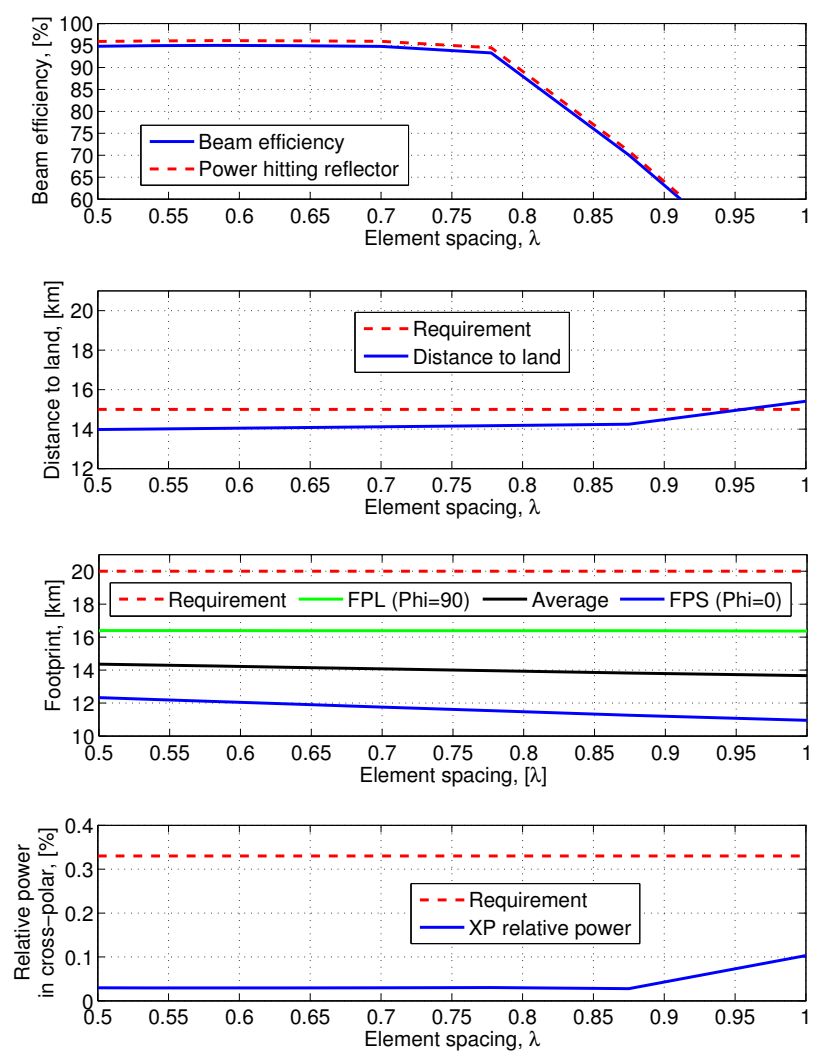

Figur 4. Radiometer characteristics as function of the FPA element spacing ( $\left.d_{\mathrm{el}}\right)$ for the case of $L_{\mathrm{x}}=7 \lambda$, including (from top to bottom) the antenna beam efficiency (defined within the $-20 \mathrm{~dB}$ region), distance to land at which the radiometer should stop working correctly, averaged footprint and relative cross-polarization power loss in the entire region.

\section{Parametric study}

The analysis of the weighting coefficients on Fig. 3 shows that many elements are weakly excited, so the size of the initially selected sub-array can be reduced along $x$-direction. Note that in $y$-direction, the sub-array cannot be smaller, since these elements will be used to form the scanning beams. We have, therefore, performed a parametric study by looking into the smaller arrays sizes, as well as different values of the element separation distances.

1) Inter-element separation distance: Fig. 2 and Fig. 4 present the first set of the results obtained for the array $7 \lambda \times 14 \lambda$ and constrained beamformer - that illustrate the effect of the element separation distance $d_{\mathrm{el}}$ on the optimized weights and corresponding aperture-field distribution of the reflector. As observed, the most dense FPA provides a very fine sampling of the array aperture field, resulting in the well-behaved illumination of the reflector, whereas the field produced by the most sparse array with $1 \lambda$-spaced elements exhibits the grating lobes. The importance of the array density can be also seen from the computed radiometer parameters that are shown on Fig. 4 as function of $d_{\mathrm{el}}$. It is interesting 


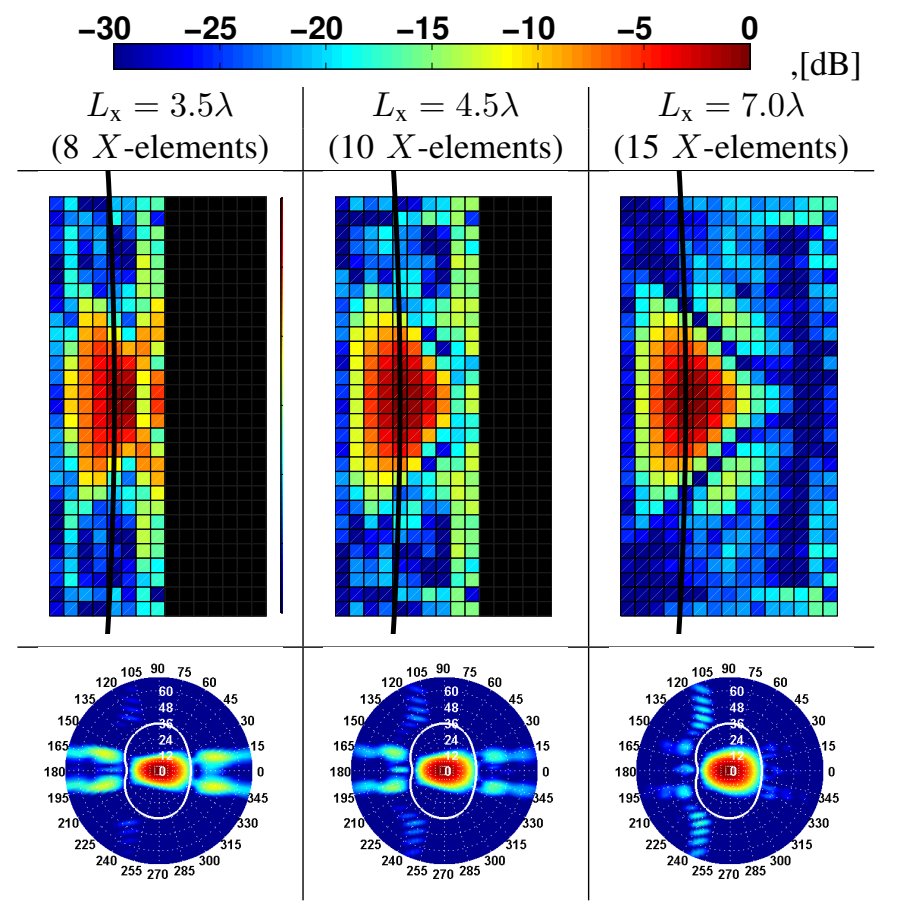

Figur 5. Effect of the array size $L_{\mathrm{x}}$ on (top) the optimized amplitude weights of the FPA sub-array elements for the centre beam, as determined for the customized beamformer maximizing the beam efficiency (@ $-20 \mathrm{~dB}$ ) with constraints on the side-lobe and cross-polarization levels towards the coastal region, and (bottom) the resultant illumination patterns of the reflector antenna. The distance between the array elements is fixed to $d_{\mathrm{el}}=0.5 \lambda$.

to see that the beam efficiency and cross-polarization power are affected most when the array becomes sparse $\left(d_{\mathrm{el}}>0.7 \lambda\right)$ - because the array aperture field gets under-sampled and the grating lobes start to appear -, while the minimum distance from land remains small almost over the entire region of $d_{\mathrm{el}}$ (and within the required value of $15 \mathrm{~km}$ ) thanks to the low side-lobes in the coastal region that are forced by the beamformer.

2) Array size: The second set of the parametric study results is illustrated on Fig. 5 and Fig. 6, that show the effect of the sub-array size along $x$-direction, for the case of $d_{\mathrm{el}}=0.5 \lambda$. These results demonstrate that all the radiometer parameters are sensitive to change of the array size, and their values degrade when it becomes smaller. This observation is expected, since the larger arrays have more degrees of freedom that the smaller ones. In general, the minimum size of the array along $\mathrm{x}$-direction should be $\sim 4.9 \lambda$ to realize the beam efficiency higher than $\sim 91 \%$ with the distance to coast according to the requirements. For $d_{\mathrm{el}}=0.7 \lambda$, this would corresponds to $8 \times 21 \times 2$ elements in total for the center sub-array. Interestingly enough, the beam efficiency of twice larger sub-array $\left(L_{\mathrm{x}}=9 \lambda\right)$ would be only a few percent higher $(\sim 96 \%)$ with the similar values of other considered radiometer parameters.

\section{CONCLUSiOns}

Table I summarizes the $X$-band performance parameters of the pushbroom radiometer that employs a torus reflector
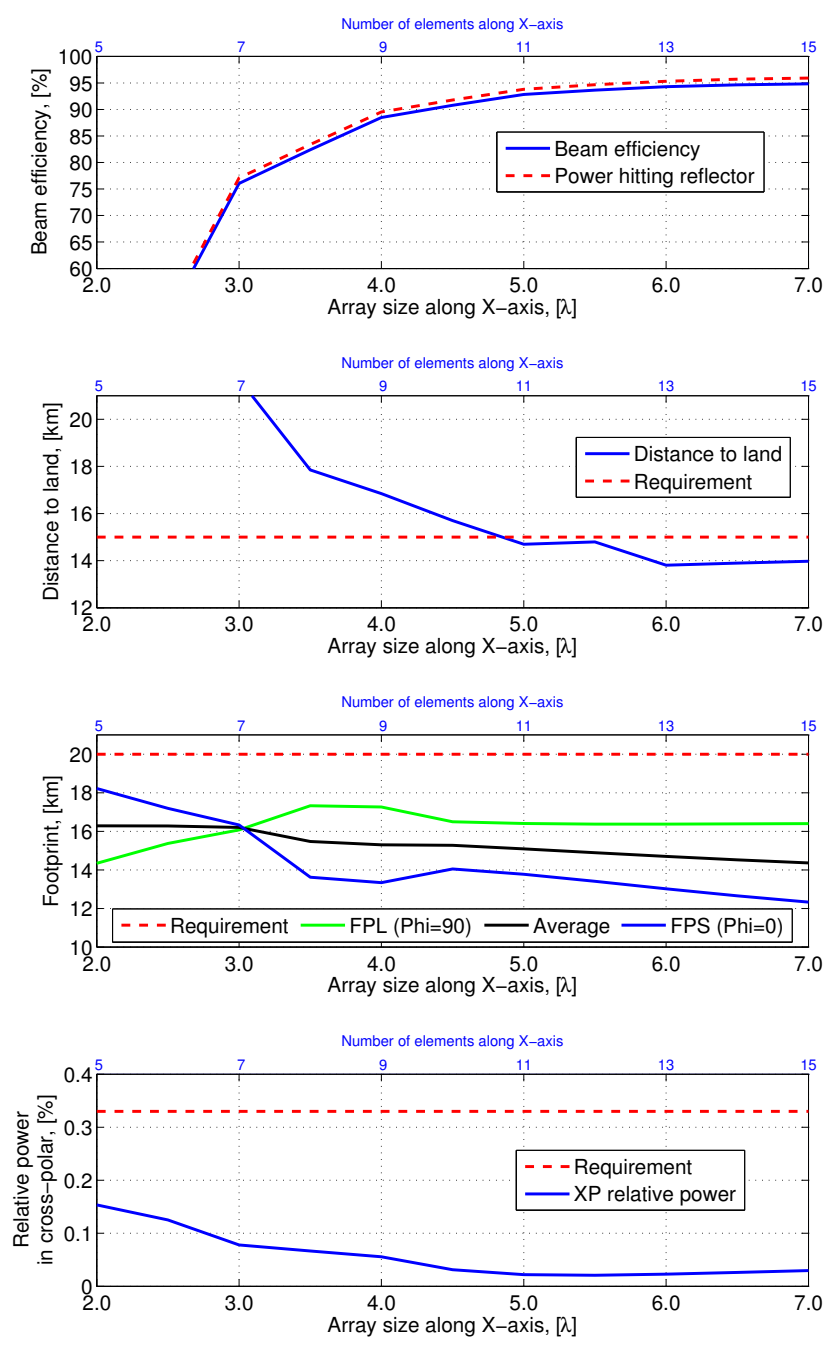

Figur 6. Radiometer characteristics as function of the number of elements (array size) alone $\mathrm{X}$-axis for the case of $d_{\mathrm{el}}=0.5 \lambda$, including (from top to bottom) the antenna beam efficiency (defined within the $-20 \mathrm{~dB}$ region), distance to land at which the radiometer should stop working correctly, averaged footprint and relative cross-polarization power loss in the entire Earth region.

Tabell I

RADIOMETER CHARACTERISTICS FOR DIFFERENT FPAS

\begin{tabular}{|l|c|c|c|c|}
\hline & $\begin{array}{c}\text { Gauss. } \\
\text { feed } \\
\text { model }\end{array}$ & $\begin{array}{c}\text { FPA with } \\
\text { CFM-BF } \\
15 \times 29 \times 2 \\
\text { elem. } \\
d_{\mathrm{el}}=0.5 \lambda\end{array}$ & $\begin{array}{c}\text { FPA with } \\
\text { Cust-BF } \\
15 \times 29 \times 2 \\
\text { elem. } \\
d_{\mathrm{el}}=0.5 \lambda\end{array}$ & $\begin{array}{c}\text { FPA with } \\
\text { Cust-BF } \\
8 \times 21 \times 2 \\
\text { elem. } \\
d_{\mathrm{el}}=0.7 \lambda\end{array}$ \\
\hline Beam efficiency [\%] & 84.2 & 85.1 & 94.9 & 92.0 \\
\hline $\begin{array}{l}\text { XP-power, [\%] } \\
(<0.33 \% \text { is req. })\end{array}$ & 0.39 & 1.01 & 0.03 & 0.02 \\
\hline $\begin{array}{l}\text { Dist. to land, [km] } \\
\text { (<15 km is req.) }\end{array}$ & 87.8 & 116.6 & 14.0 & 15.9 \\
\hline Beam width, [deg] & 0.600 & 0.351 & 0.512 & 0.538 \\
\hline $\begin{array}{l}\text { Footprint (FP), [km] } \\
\text { (<20 km is req.) }\end{array}$ & 16.9 & 10.5 & 14.4 & 14.9 \\
\hline FP ellipticity & 1.38 & 2.14 & 1.33 & 1.22 \\
\hline
\end{tabular}

antenna with the 5-m diameter projected aperture for different types of FPAs (i.e conventional FPAs of horns in one-horn-perbeam-configuration that are herein represented by Gaussian 
beams, and dense FPAs of tapered-slot antenna elements with different beamforming scenarios). As expected, dense FPAs have obvious benefits in achieving the required minimum distance to coast and footprint roundness, while meeting all other radiometer requirements. The minimum size of the FPA sub-array has been found to be $8 \times 21$ elements (for each polarization) with the inter-element separation distance in the order of $d_{\mathrm{el}}=0.7 \lambda$, for the considered initial model of the array.

\section{ACKNOWLEDGMENT}

The present work has been carried out in the framework of the "Advanced Multi-Beam Radiometers" project that is a collaborative effort between TICRA, DTU-Space (Denmark), HPS (Germany) and Chalmers, funded by European Space Agency (ESA). The toroidal push-broom reflector antenna used for our study has been designed by TICRA. The authors would like to acknowledge the Swedish Research Council for providing partial support to this work through the VR project grant.

\section{REFERANSER}

[1] (2013, Sep.). [Online]. Available: http://earthobservatory.nasa.gov/Features/EO1/eo1_2.php

[2] P. Nielsen, K. Pontoppidan, J. Heeboell, and B. L. Stradic, "Design, manufacture and test of a pushbroom radiometer," in Antennas and Propagation, 1989. ICAP 89., Sixth International Conference on (Conf. Publ. No.301), Coventry, United Kingdom, Apr. 1989, pp. 126-130.

[3] R. Hoferer and Y. Rahmat-Samii, "RF characterization of an inflatable parabolic torus reflector antenna for space-borne applications," IEEE Trans. Antennas Propag., vol. 46, no. 10, pp. 1449-1457, Oct. 1998.

[4] M. V. Ivashina, O. Iupikov, R. Maaskant, W. A. van Cappellen, and T. Oosterloo, "An optimal beamforming strategy for wide-field surveys with phased-array-fed reflector antennas," IEEE Trans. Antennas Propag., vol. 59, no. 6, pp. 1864-1875, Jun. 2011.

[5] W. A. van Cappellen and L. Bakker, "APERTIF: Phased array feeds for the Westerbork synthesis radio telescope," in IEEE International Symposium on Proc. Phased Array Systems and Technology (ARRAY), Boston, Oct. 2010, pp. 640-647.

[6] K. F. Warnick, "High efficiency phased array feed antennas for large radio telescopes and small satellite communication terminals," Gothenburg, Sweden, Apr. 2013, pp. 448-449.

[7] S. G. Hay, J. D. O'Sullivan, J. S. Kot, C. Granet, A. Grancea, A. R. Forsyth, and D. H. Hayman, "Focal plane array development for ASKAP (australian SKA pathfinder)," Edinburgh, UK, Nov. 2007, pp. 1-5.

[8] C. Cappellin, K. Pontoppidan, P. Nielsen, N. Skou, S. S. Sbjrg, A. Ihle, D. Hartmann, M. Ivashina, O.Iupikov, and K. v. t Klooster, "Novel multi-beam radiometers for accurate ocean surveillance," The Hague, The Netherlands, Apr. 2014, pp. 1-4.

[9] C. Kidd, "Radio frequency interference at passive microwave earth observation frequencies," Int. Journal of Remote Sensing, vol. 27, no. 18, pp. 3853-3865, Sep. 2006. 